\title{
Extension of Some Results on Forced Nonlinear Oscillations $\left(^{*}\right)\left(^{* *}\right)$. \\ GaBRIEle VILLARI (Firenze)
}

A Gaetano VillaRr, mio padre, per $i$ suoi sessanta anni

Sunto. - Si eonsidera l'equazione

$$
\ddot{x}+f(x, \dot{x}) \dot{x}+g(x)=e(t)=e(t+T) .
$$

Si danno due teoremi per l'esistenza di soluzioni di periodo $T$ basandosi su una applicazione del teorema di punto fisso di Brouwer.

\section{Introduction.}

In the present paper we consider generalized Liénard's differential equation

$$
\ddot{x}+f(x, \dot{x}) \dot{x}+g(x)=e(t)=e(t+T)
$$

We give sufficient conditions for the existence of $T$-periodic solutions.

Results of this kind have been obtained by N. Levinson [1], C. LaNGenHop [2], Z. Opiat [3], R. Rueissig [4] and Gamtano Villari [5].

The technique used in the demonstration is analogous to that used in the papers mentioned above, and is based on an application of Brouwer's fixed point theorem.

When equation (1) is written out in the form of the system

$$
\left\{\begin{array}{l}
\dot{x}=y \\
\dot{y}=-f(x, y) y-g(x)+e(t)
\end{array}\right.
$$

in the plane $t=$ constant we may locate a finite simply connected region $V$, defined by one or more simple line arcs. These lines are for the most part trajectories of autonomous systems or projections on the plane $t=$ constant of solutions of nonautonomous systems which, for convenience, we shall also continue to refer to as trajectories.

Let $\omega$ be the transformation which associates the projection on the plane $t=$ constant of the point obtained by following for time $T$ the integral curve of

(*) Entrata in Redazione il 13 agosto 1983.

(**) This work was carried out under the auspices of G.N.A.F.A.C.N.R. 
system (2) which passes through $P$, with the generic point $P$ of the plane $t=$ constant. One may prove that the region $V$ has the property of being transformed by $\omega$ into one of its parts.

The Brouwer theorem, therefore, assumes the existence of a fixed point for the transormation $\omega$, and hence, for the periodicity of $e(t)$, one has the existence of a periodic solution for system (2) and thus for equation (1).

In this paper we give two theorems for the existence of periodic solutions based on two different ways of defining a suitable region $V$. The type of construction used in Theorem 2 may also be applied relatively to the autonomous equation

$$
\ddot{x}+f(x, \dot{x}) \dot{x}+g(x)=0
$$

obtaining additionally in this case a theorem for the existence of periodic solutions.

If in equation (1) we consider the function $f$ dependent only on $x$, we obtain

$$
\ddot{x}+f(x) \dot{x}+g(x)=e(t)=e(t+T) .
$$

This type of equation has been the object of study in the last few years, because in this case one may use a different technique.

J. MaWHIN [6] generalizing a result by C. LAZEeR [7], has demonstrated the existence of a periodic solution for equation (3) with only the hypothesis of continuity relative to $f(x)$. The demonstration is based on Schauder's fixed point theorem. This result has been expanded on by R. ReIssig [8], [9], M. Martelli [10] and many other authors.

It does not, however, seem possible, as Mawhin and Martelli assert in the abovementioned papers, to extend these results to equation (1) without adding further hypotheses on $f(x, \dot{x})$ besides that on continuity; this does not seem convenient considering that the hypothesis on $g(x)$ are heavier with respect to the works already considered.

In the final part of the paper, we show that the results obtained are more general then their predecessors, and compare the differences between the hypotheses relevant to a study of equation (3).

1. - THEOREM 1. - Let $f: \boldsymbol{R}^{2} \rightarrow \boldsymbol{R}, y: \boldsymbol{R} \rightarrow \boldsymbol{R}$ and $e: \boldsymbol{R} \rightarrow \boldsymbol{R}$ be such that:

(1) $f$ and $g$ are continuous and locally Lipschitz continuous functions;

(2) $e(t)$ is continuous and periodic, $e(t)=e(t+T) \forall t, e \leqslant e(t) \leqslant E$;

(3) there exist $a, b, M>0$ with $b>a$ such that

$$
\begin{gathered}
f(x, y)>-M \quad \text { if }|x|<a, \quad|y|<+\infty \\
x>b, \quad y<-a \\
x<-a, \quad y>x
\end{gathered}
$$


(4)

$$
\begin{aligned}
f(x, y) y+g(x) \geqslant E & \text { if } x>a \quad y \geqslant 0 \\
f(x, y) y+g(x) \leqslant e & \text { if } x<-a \quad y \leqslant 0 \\
f(x, y)+f(x, z) \geqslant k>0 & \text { if } x>b, \quad y \geqslant z>a \\
& x<-a, \quad y \leqslant z<-a
\end{aligned}
$$

(6) there exists $\Phi(x)$ such that

$$
f(x, y) \geqslant \Phi(x) \quad \text { if } a \leqslant x \leqslant b \quad y<-a_{1} \leqslant-a
$$

and

$$
\int_{a}^{b} \Phi(x) d x>4 a N+1
$$

where $N=M+H, H>0$ is such that

$$
g(x)-e(t) \geqslant-H \quad \text { if }|x|<a \quad 0 \leqslant t \leqslant T
$$

and $a_{1}>a$ is such that

$$
0<\frac{E-e}{a_{1}} \leqslant \frac{k}{3}
$$

Then there exists a $T$-periodic solution of equation (1).

Proof. - We write the equation

$$
\ddot{x}+f(x, \dot{x}) \dot{x}+g(x)=e(t)
$$

in the form of the system

$$
\left\{\begin{array}{l}
\dot{x}=y \\
\dot{y}=-f(x, y) y-g(x)+e(t) .
\end{array}\right.
$$

We assume $E>e$; if indeed $E=e$, then $e(t)$ is a constant, system (1.1) is autonomous and hypotheses (4) guarantee the existence of a stationary point and thus of a periodic solution.

In the plane-region bounded by $x \geqslant b, y \geqslant 0$ we consider the autonomous system

$$
\left\{\begin{array}{l}
\dot{x}=y \\
\dot{y}=-f(x, y) y-g(x)+E
\end{array}\right.
$$


It follows from assumption (4) that for such a system it is

$$
\dot{y} \leqslant 0 \text {. }
$$

If we assume, as in [5], $\dot{y} \leqslant-k / 3<0$, considering a point $P_{0}\left(x_{0}, y_{0}\right)$, having coordinates $x_{0}$ and $y_{0}$ with $x_{0} \geqslant b$ and $y_{0}>0$, the positive half-trajectory of system (1.2) which passes through the point $P_{\mathbf{0}}$ crosses in a finite time the $x$-axis at a point $P_{1}\left(x_{1}, 0\right)$ and it is $x_{1}-x_{0} \leqslant \frac{3}{2}\left(y_{0}^{2} / k\right)$.

Now we consider points such that for system (1.2)

$$
-\frac{k}{3} \leqslant \dot{y} \leqslant 0
$$

and assume, for convenience, that (1.3) holds for every point of the region bounded by $x \geqslant b, y \geqslant 0$.

Let $E-e>0$ and let

$$
\delta=\frac{E-e}{4} .
$$

We consider the function $e(t)$ in the interval $[0, T]$. Let $t_{0}$ be a point of the open interval $(0, T)$ such that $e\left(t_{0}\right)=e+2 \delta$. Recalling the uniform continuity of $e(t)$ which is due to periodicity one may choose $h>0$ such that from $\left|t-t_{0}\right| \leqslant h$ it follows that $\left|e(t)-e\left(t_{0}\right)\right|<\delta$.

Divide the interval $[0, T]$ into two sets $T_{1}$ and $T_{2}$ :

$$
\begin{aligned}
& {[0, T]=T_{1} \cup T_{2} } \\
T_{1}= & \left(t_{0}-h, t_{0}+h\right) \\
T_{2}= & {\left[0, t_{0}-h\right] \cup\left[t_{0}+h, T\right] . }
\end{aligned}
$$

Let $\varphi: \boldsymbol{R} \rightarrow \boldsymbol{R}, \Psi: \boldsymbol{R} \rightarrow \boldsymbol{R}$ and $v: \boldsymbol{R} \rightarrow \boldsymbol{R}$ be such that $\varphi, \Psi$ and $v$ are $T$-periodic functions and

$$
\begin{aligned}
& \varphi(t)= \begin{cases}E-\delta & \text { if } t \in T_{1} \\
E & \text { if } t \in T_{2}\end{cases} \\
& \Psi(t)= \begin{cases}e+\delta & \text { if } t \in T_{1} \\
e & \text { if } t \in T_{2}\end{cases} \\
& v(t)= \begin{cases}-\delta & \text { if } t \in T_{1} \\
0 & \text { if } t \in T_{2}\end{cases}
\end{aligned}
$$


We have $\Psi(t)<\varphi(t)$

$$
\Psi(t) \leqslant e(t) \leqslant \varphi(t) \quad \forall t
$$

One may choose $a_{1}>a$ such that

$$
0<\frac{E-e}{a_{1}} \leqslant \frac{k}{3}
$$

Now we may consider the system

$$
\begin{cases}\dot{x}=y & x \geqslant b \\ \dot{y}=v(t) & y>0\end{cases}
$$

LEMrus 1. - When the time $t_{0}-h$ and the point $P_{0}\left(x_{0}, y_{0}\right)$ are fixed, the positive halftrajectory of system (1.5) which passes through the point $P_{\mathbf{0}}$ at the time $t_{0}-h$, crosses in a finite time the $x_{\text {-axis at a point }} P_{1}\left(x_{1}, 0\right)$ and it is possible to give a bound for the the distance $x_{1}-x_{0}$ which does not depend on $x_{0}$.

The proof of this Lemma and the proofs of Lemma 2 and Lemma 3 which follow are in the Appendix at the end of this paper.

REMaRK 1.1. - We consider the system

$$
\begin{cases}\dot{x}=y & x \geqslant b \\ \dot{y}=-f(x, y) y-g(x)+\varphi(t) & y \geqslant 0\end{cases}
$$

From a comparison with the system (1.5) it follows that the positive half-trajectory of system (1.6) which passes through the point $P_{0}$ at the time $t_{0}-h$ has an upper boundary which is the trajectory of system (1.5) which passes through the point $P_{0}$ at the same time. So the considered trajectory of system (1.6) crosses in a finite time the $x$-axis at a point $\hat{P}(\hat{x}, 0)$. Clearly $\hat{x}-x_{0} \leqslant x_{1}-x_{0}$.

We consider the point $B\left(x_{B}, a_{1}\right)$, with $x_{B}>x_{0}$.

Based on Lemma 1 one may see that the positive half-trajectory of system (1.5) which passes through the point $B$ at the time $t_{0}-h$, meets the point $C\left(x_{C}, a\right)$, with $x_{C}>x_{B}$, and then crosses at the time $\hat{t}$ the $x$-axis at a point $D\left(x_{D}, 0\right)$ with $x_{D}>x_{C}$.

In the half-plane $y \leqslant 0$ we consider the autonomous system

$$
\left\{\begin{array}{l}
\dot{x}=y \\
\dot{y}=-f(x, y) y-g(x)+e
\end{array}\right.
$$

Recalling that $f(x, y)$ has a lower bound in the plane region bounded by $x>a$ and $y<-a$, it is easy to prove that the positive half-trajectory of system (1.7) which passes through the point $F\left(x_{D},-a_{1}\right)$ crosses in a finite time the axis $x=x_{0}$ in $y<0$. 
376

G. VILLARI: Extension of some results on forced nonlinear oscillations

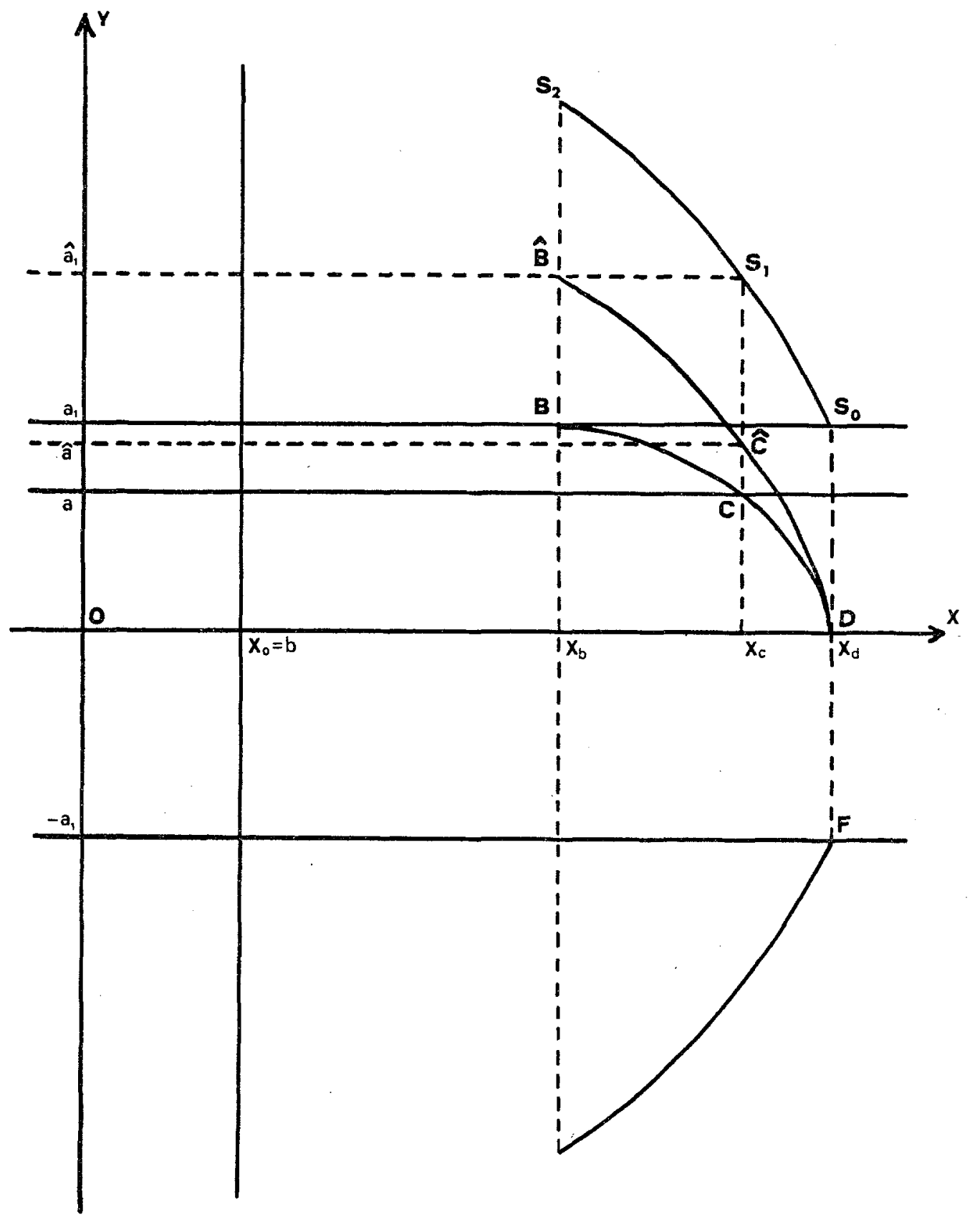

Fig. 1. 
Let $\hat{y}(x)$ be the symmetric line, with respect to the $x$-axis, of a trajectory of system (1.7).

Such a line satisfies the equation

$$
\hat{y}^{\prime}=f\left(x_{1},-\hat{y}\right)-\frac{g(x)-e}{\hat{y}}
$$

which comes from

$$
y^{\prime}=-f(x, y)-\frac{g(x)-e}{y}
$$

a consequence of system (1.7), where one makes the sobstitution

$$
\hat{y}=-y
$$

We consider the point $S_{0}\left(x_{D}, a_{1}\right)$, which is the symmetric point of $F$.

The symmetric line of the trajectory of system (1.7) which passes through $F$, passes through $D_{0}$ and meets the point $S_{1}\left(x_{C}, y\right)$ and then the point $S_{2}\left(x_{B}, y\right)$.

LEMMA 2. - The length of the segment $S_{2} B$ has an upper bound which does not depend on $x_{0}$.

A consequence of Lemma 2 is the following

LEMrMa 3. - One may choose $y_{0}>a_{1}$ such that

1) The positive half-trajectory of system (1.5) which passes through the point $P_{0}\left(b, y_{0}\right)$ at the time $\hat{t}$ erosses in a finite time the $x$-axis at a point $\hat{P}(\hat{x}, 0)$.

2) The positive half-trajectory of system (1.7) which passes through the point $\hat{Q}\left(\hat{x}, a_{1}\right)$ crosses in a finite time the axis $x=b$ at a point $Q_{0}\left(b,-y_{Q}\right)$ and

$$
0<y_{Q}<y_{0}
$$

3) These assumptions hold for every $y>y_{0}$, and the choice of the time $\hat{t}$ has no infuence.

REMARK 1.2. - The result of Lemma 3 still holds if we consider the axis $x=-a$ instead of the axis $x=b$, the system

$$
\begin{array}{ll}
\dot{x}=y & x \leqslant-a \\
\dot{y}=-f(x, y) y-g(x)+\Psi(t) & y<0
\end{array}
$$


instead of the system (1.6) and the system

$$
\begin{cases}\dot{x}=y & x \leqslant-a \\ \dot{y}=-v(t) & y<0\end{cases}
$$

instead of the system (1.5).

We assume that for every point of the region bounded by $x \leqslant-a, y \leqslant 0$ it is

$$
0 \leqslant \dot{y} \leqslant \frac{k}{3} .
$$

Such an assumption is made for convenience and is analogous with (1.3).

In the half-plane $y \geqslant 0$ we consider the autonomous system (1.2) instead of the system (1.7).

Clearly we consider the symmetric lines, with respect to the $x$-axis, of the trajectories of system (1.2).

The result still holds for every $y<-y_{0}$ with $-y_{0}<-a_{1}$.

Again, the choice of time has no influence.

It is possible to choose a constant $H>0$ such that

$$
\begin{aligned}
g(x)-e(t) \geqslant-H \quad \text { if }|x|<a \\
0 \leqslant t \leqslant T .
\end{aligned}
$$

Let $N=M+H$.

It is trivial to see that if the positive half-trajectory of system (1.1) which passes through a point $R(-a, \bar{y})$ of the axis $x=-a$, with $\bar{y}>1$, at the time $t$, crosses in a finite time the axis $x=a$ it does it at a point $\hat{R}(a, y)$ with $y<\bar{y}+2 a N$. Such a part of the trajectory is bounded on the upper side by the segment whose extremes are $R$ and $\bar{R}(a, \bar{y}+2 a N)$.

The same result holds if we consider a trajectory of system (1.7) and the points $R_{1}(a,-\bar{y})$ and $\bar{R}_{1}(-a,-(\bar{y}+2 a N))$.

Based on hypothesis (6) one may choose $Z>0$ such that

$$
-\int_{a}^{b} \Phi(x) d x+\frac{G}{Z}(b-a)<-4 a N
$$

where

$$
G=\max \{g(x)-e, a \leqslant x \leqslant b\}
$$

Let

$$
\hat{\Phi}=\max \{\Phi(x), \quad a \leqslant x \leqslant b\},
$$

one may choose $\hat{Z}$ such that

$$
\hat{Z}>Z+(b-a) \cdot\left[\hat{\Phi}+\frac{G}{Z}\right] .
$$


Based on (1.10), (1.11) and hypothesis (6) one can see that the positive half-trajectory of system (1.7) which passes through a point $P_{1}(b, y)$ of the axis $x=b$, with $y \leqslant-\hat{Z}$, crosses in a finite time the axis $x=a$ at a point $P_{2}(a, y)$, with $y$ such that

$$
-(\hat{Z}-4 N)<y<-Z
$$

Finally we may locate in the phase-plane a suitable region $V$ for which it is possible to apply the method which is described in the introduction.

With reference to the figure, one may choose $\hat{Z}$ such that it satisfies the assumption (1.11), where the value $Z$ satisfies the assumption (1.10) and is greater than $y_{0}>a_{1}$ with $y_{0}$ as in Lemma 3 . Here we assume that $y_{0}$ satisfies Lemma 3 also for the axis

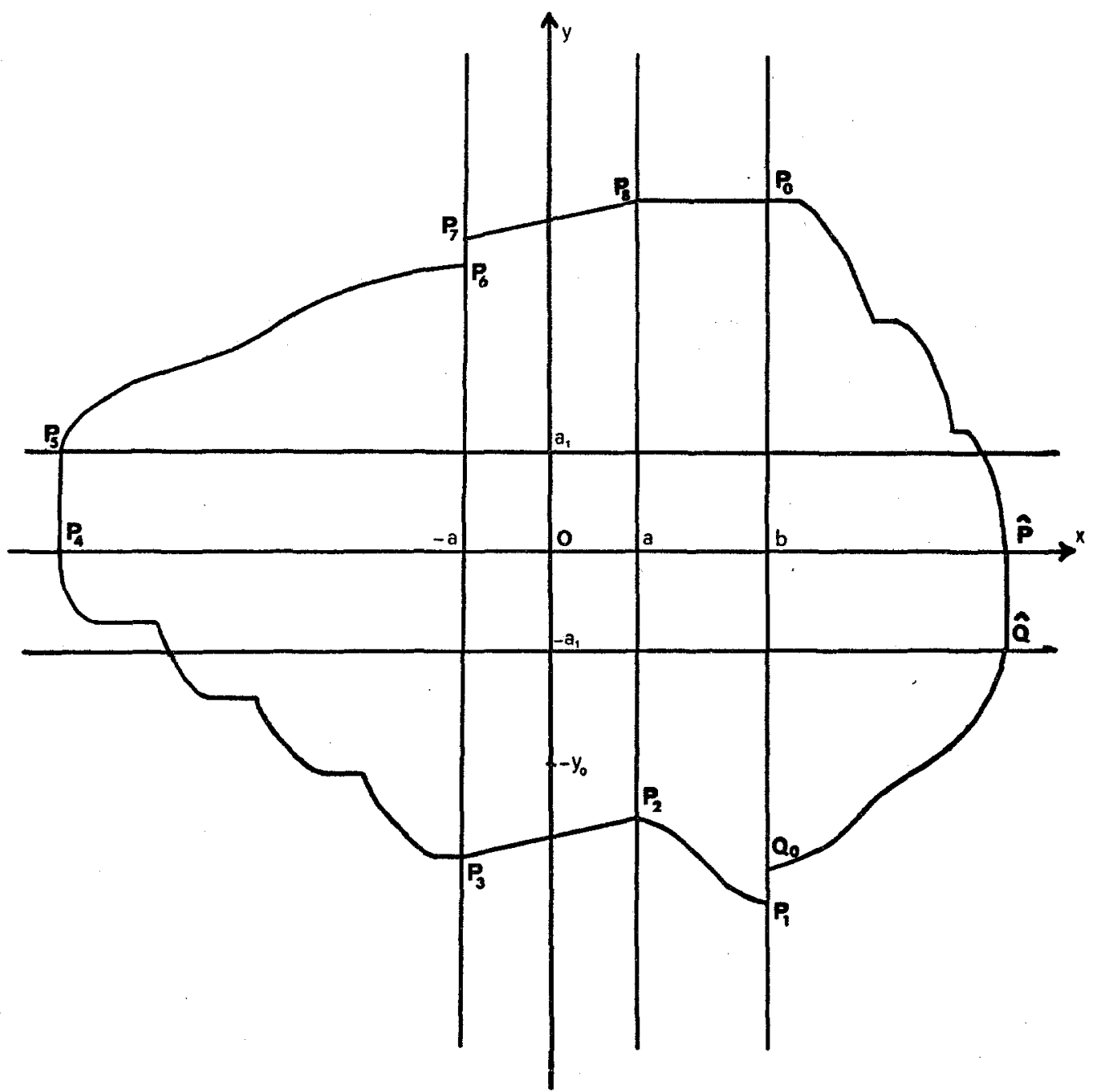

Fig. 2. 
$x=-a$, which is clearly not restrictive on a possible enlargement of the above choice of $y_{0}$.

The point $P_{1}$ has coordinates $x=b$ and $y=-\hat{Z}$. The point $P_{2}$ has coordinates $x=a$ and $y=-\bar{Z}$.

Based on (1.12) it is $-(\hat{Z}-4 a N)<-\bar{Z}<-Z<-y_{0}$.

The point $P_{3}$ has coordinates $x=-a$ and $y=-\bar{Z}-2 a N, P_{4}$ and $P_{5}$ have the same abscissa. $P_{6}$ has coordinates $x=a$ and $y<\bar{Z}+2 a N . P_{7}$ has coordinates $x=-a, y=\bar{Z}+2 a N . P_{8}$ has coordinates $x=a$ and $y=\bar{Z}+4 a N$. $P_{0}$ has coordinates $x=b$ and $y=\bar{Z}+4 a N . \hat{P}$ and $\hat{Q}$ have the same abscissa.

At last, $Q_{0}$ has coordinates $x=b$ and $y>-\bar{Z}-4 a N>-\hat{Z}$. The line $P_{1}-Q_{0}$ is formed in this way:

$$
\overline{P_{2} P_{3}}, \quad \overline{P_{4} P_{5}}, \quad \overline{P_{7} P_{8}}, \quad \overline{P_{8} P_{0}} \text { and } \overline{\hat{P} \hat{Q}} \text { are segments. }
$$

$\widehat{Q Q}_{0}$ and $\widehat{P_{1} P_{2}}$ are trajectories of the autonomous system (1.7). $\widehat{P}_{5} P_{8}$ is a trajectory of the autonomous system (1.2). $\overparen{P_{0} \hat{P}}$ is a trajectory of system (1.5) with the initial condition $t_{0}+h . \widehat{P}_{3} P_{4}$ is a trajectory of system (1.9) with the initial condition $t_{0}+h$.

Notice that the lines $P_{0} Q_{0}$ and $P_{3} P_{7}$ are based on Lemma 3 , while the line $P_{1} P_{2}$ is based on hypothesis $(6)$. One may verify that it is possible to apply the Brouwer's fixed point theorem to the region $V$ which is bounded by the line $P_{1}-Q_{0}$.

We project the region $V$ on the plane $t=t_{0}-h$. Let $\omega$ be the transformation which associates the projection on the plane $t=t_{0}-h$ of the point obtained by following for time $T$ the positive half-trajectory of system (1.1) which passes though $P$ at the time $t_{0}-h$, with the generic point $P$ of the plane $t=t_{0}-h$; one may prove that the region $V$ has the property of being transformed by $\omega$ in one of its parts.

The result is trivial for the parts of the region $V$ which are bounded by segments and by arcs of trajectories of system (1.2) and (1.7).

In fact the trajectories of system (1.7) are in the half-plane $y \leqslant 0$, while the trajectory of system (1.2) is in the half-plane $y \geqslant 0$.

With respect to the parts of $V$ which are bounded by the lines $P_{0} \widehat{P}$ and $P_{3} P_{4}$, a direct proof is necessary. For simplicity we examine in detail only the region bounded bounded by the line $P_{0} \hat{P}$, the axis $x=b$ and the axis $y=0$. It is necessary to prove that a generic point $P$ of this region is transformed by $\omega$ into a point $P^{\prime}$ which is in the same region if it is in the half-plane $y \geqslant 0$. We may examine only the points of the trajectory $P_{0} \hat{P}$, because two trajectories of system (1.5) which pass at the same time, through two points with the same abscissa cannot cut each other, having the same behaviour of autonomous trajectories.

A comparison between system (1.1) and system (1.5) shows that the positive halftrajectory of (1.1) which passes through a point of the considered region is bounded by the corresponding trajectory of system (1.5) which passes at the same time through the same point. 
We suppose, for simplicity, that the positive half-trajectory of system (1.5) which passes through the point $P_{0}(\bar{Z}+4 a N, b)$ at the time $t_{0}+h$, crosses in a time $2 T$ the $x$-axis at a point $\hat{P}$. This situation is described in the figure.

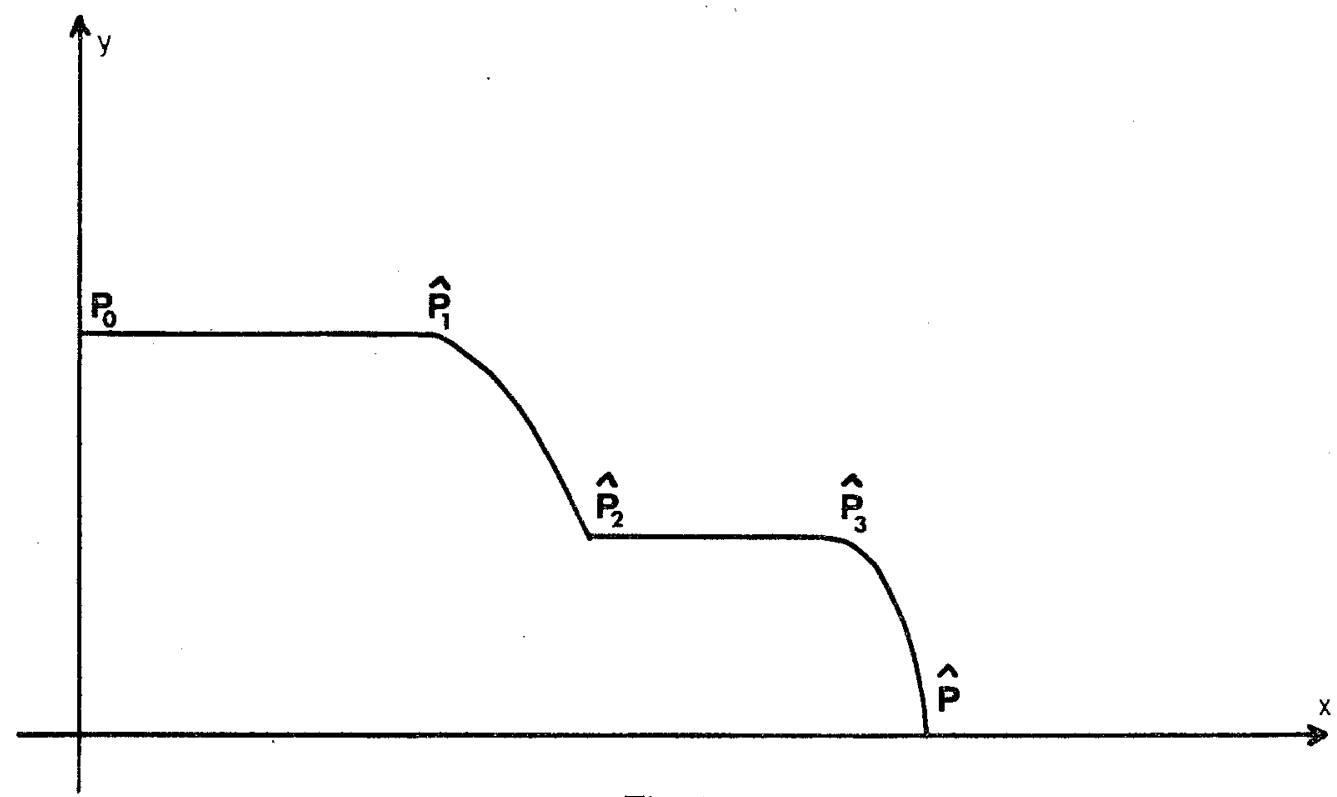

Fig. 3.

Consider only the line $P_{0} \hat{P}_{2}$. If we choose a positive half-trajectory of system (1.5) which passes through any point of the line $P_{0} \hat{P}_{2}$ at the time $t_{0}-h$, such a trajectory does not cross the line $P_{0} \hat{P}$ during the time $T$. Notice that the result is true only for the initial time $t_{0}-h$. This result holds for the whole line $P_{0} \hat{P}$ and the proof is analogous for the line $P_{3} P_{4}$.

Thus the region $V$ has the property of being transformed by $\omega$ into one of its parts and this completes the proof of the Theorem.

Remark 1.3. - Assumption (1.3) is not restrictive. In the general case the line $P_{0} \hat{P}$ may be construct with trajectories of the autonomous system (1.2) and trajectories of system (1.5) with the initial condition $t_{0}+h$.

One may then construct the line $P_{3} P_{4}$ in the same way, using trajectories of the autonomous system (1.7) and system (1.9) with the initial condition $t_{0}+h$.

RemarK 1.4. - We just note that in the hypothesis (6) the assumptions

$$
\begin{gathered}
f(x, y) \geqslant \Phi(x) \quad \text { if } a \leqslant x \leqslant b \quad y<-a_{1} \leqslant-a \\
\int_{a}^{b} \Phi(x) d x>4 a N+1
\end{gathered}
$$


may be replaced by the assumptions

$$
\begin{gathered}
f(x, y) \geqslant \Phi(x) \quad \text { if }-b \leqslant x \leqslant-a, \quad y>a_{1} \geqslant a \\
\int_{-b}^{-a} \Phi(x) d x>4 a N+1
\end{gathered}
$$

provided that one makes the corresponding changes in the hypotheses (3) and (5), namely

$$
\begin{array}{ll}
x>a & \text { instead of } x>b \\
x<-b & \text { instead of } x<-a
\end{array}
$$

REMark 1.5. - Recalling the construction of the plane-region $V$ it is clear that Theorem 1 may be invoked to assert that

Every solution of the equation

$$
\ddot{x}+f(x, \dot{x}) \dot{x}+g(x)=e(t)
$$

is bounded for $t>0$.

This result is still true if the function $e(t)$ is not a periodic function.

If $e(t)$ is a constant, or if

$$
\lim _{t \rightarrow+\infty} e(t)=E
$$

the hypothesis (4) is replaced by

$$
\begin{array}{ll}
f(x, y) y+g(x) \geqslant E+k & \text { if } x \geqslant b, \quad y \geqslant 0 \\
f(x, y) y+g(x) \leqslant E-k & \text { if } x \leqslant-a, \quad y \leqslant 0
\end{array}
$$

where $k>0$ is a constant.

2. - With the same technique, and using the previous results, one may state the following

THEOREM 2. - Let $f: \boldsymbol{R}^{2} \rightarrow \boldsymbol{R}, g: \boldsymbol{R} \rightarrow \boldsymbol{R}$ and $e: \boldsymbol{R} \rightarrow \boldsymbol{R}$ such that:

(1) $f$ and $g$ are continuous and locally Lipsehitz continuous functions;

(2) $e(t)$ is continuous and periodic, $e(t)=e(t+T) \forall t, e \leqslant e(t) \leqslant E$;

$\left(3^{\prime}\right)$ there exist $a, M>0$ such that

$$
\begin{aligned}
& f(x, y)>-M \quad \text { if } x<a, y>a \\
& x>-a, y<-a
\end{aligned}
$$


(4)

$$
\begin{array}{cl}
f(x, y) y+g(x) \geqslant E \quad & \text { if } x>a, \quad y \geqslant 0 \\
f(x, y) y+g(x) \leqslant e \quad \text { if } x<-a, \quad y \leqslant 0 ; & \lim _{x \rightarrow-\infty} f(x, E-g(x))>g^{\prime}(x)+1 .
\end{array}
$$

Then there exists a $T$-periodic solution of equation (1).

Proof. - Based on hypothesis $\left(5^{\prime}\right)$, there exists $\bar{x}<-a$ such that

$$
f(x, E-g(x))>g^{\prime}(x)+1
$$

if $x \leqslant \bar{x}$.

We consider the point $P_{0}$ having coordinates $\bar{x}$ and $E-g(\bar{x})$. Clearly $E-g(\bar{x})>0$. Based on hypothesis $\left(3^{\prime}\right)$, the positive half-trajectory of system (1.2) which passes through the point $P_{0}$ crosses in a finite time the axis $x=-a$.

Later on such a trajectory either crosses the $x$-axis, or crosses the axis $x=a$ at a point $P_{1}$ having coordinates $a$ and $y_{1}>0$. In the latter case we consider the positive half-trajectory of system (1.5) which passes through the point $P_{1}$ at the time $t_{0}+h$. Recalling Lemma 1 this trajectory crosses in a finite time the $x$-axis at a point $\boldsymbol{P}_{2}$. Now we consider the positive half-trajectory of system (1.7) which passes through the point $P_{2}$. Again based on hypothesig $\left(3^{\prime}\right)$ this trajectory crosses in a finite time the axis $x=a$. Later on such a trajectory either crneses the $x$-axis, or crosses the axis $x=-a$ at a point $P_{3}$ having coordinates $-a$ and $y_{3}<0$. In the latter case we consider the positive half-trajectory of system (1.12) which passes through the point $P_{3}$ at the time $t_{0}+h$. Again recalling Lemma 1 this trajectory crosses in a finite time the $x$-axis at a point $P_{4}$ having abscissa $x_{4}$.

If $x_{4} \geqslant \bar{x}$ we consider on the $x$-axis the point $P_{5}$ having abscissa $\bar{x}$. Then we join $P_{4}$ with $P_{5}$ and $P_{5}$ with $P_{0}$ : If $x_{4}<\vec{x}$ we consider on the axis $x=x_{4}$ the point $P_{5}$ having ordinate $E-g\left(x_{4}\right)$. Then we join $P_{4}$ with $P_{5}$ and we consider the line $P_{5} P_{0}$ having coordinates $x$ and $E-g(x)$, with $x_{4} \leqslant x \leqslant \bar{x}$.

One may verify that it is possible to apply the Brouwer's fixed point theorem to the region $V$ which is bounded by the line $P_{0}-P_{5}$.

Indeed if $x_{4} \geqslant \bar{x}$ one may prove, with the same arguments of theorem 1 , that the region $V$ has the property of being transformed by $\omega$, which is defined as in Theorem 1 , into one of its parts. In the second case, when $x_{4}<\vec{x}$, a direct proof is necessary with respect to the part of $V$ which is bounded by the line $P_{5} P_{0}$. For the points of the line $P_{5} P_{0}$ we have

$$
y(x)=E-g(x)
$$

which implies

$$
y^{\prime}(x)=-g^{\prime}(x)
$$

25 - Annali di Matematica 
One may write system (1.2) for these points and obtain

$$
\left\{\begin{array}{l}
\dot{x}=E-g(x) \\
\dot{y}=-f(x, E-g(x))(E-g(x))+E-g(x) .
\end{array}\right.
$$

It follows immediately from assumption (2.2) that

$$
y^{\prime}(x)=-f(x, E-g(x))+1
$$

It is necessary that any trajectory of system (1.2) which passes through a generic Point $P$ of the region $V$ cannot cross the line $P_{5} P_{0}$.

This implies that

$$
\begin{gathered}
-f(x, E-g(x))+1<-g^{\prime}(x) \\
f(x, E-g(x))>g^{\prime}(x)+1
\end{gathered}
$$

which is precisely assumption (2.1).

The Theorem is proved, because now it is possible to argue in a manner analogous to that of Theorem 1.

REMARK 2.1. - We suppose that $g(x)$ is differentiable, but this is not restrictive. Indeed if $g(x)$ is not differentiable we consider a differentiable function $g(x)$ such that

$$
\lim _{x \rightarrow-\infty} \frac{g(x)}{\hat{g}(x)}=1
$$

and we write assumption $\left(5^{\prime}\right)$ in the form

$$
\lim _{x \rightarrow-\infty} f(x, E-\hat{g}(x))>\hat{g}^{\prime}(x)+1 .
$$

Another way of writing assumption $\left(5^{\prime}\right)$ is the following:

$$
\text { let } \lim _{x \rightarrow-\infty} \frac{g(x)}{x^{n}}=\alpha \neq \infty \text {. }
$$

Define the function $y(x)=(-1)^{n} x^{n}$.

We consider the system (1.2) for the points having coordinates $x$ and $(-1)^{n} x^{n}$. Arguing as in the previous theorem, based on assumption (2.3), it follows that

$$
-f\left(x,(-1)^{n} x^{n}\right)-(-1)^{n} \alpha<(-1)^{n} n x^{n-1} .
$$


Hence the hypothesis $\left(5^{\prime}\right)$ may be written in the following way

$$
\begin{aligned}
& \lim _{x \rightarrow-\infty} \inf f\left(x,(-1)^{\prime} x^{n}\right)>(-1)^{n+1}\left(\alpha+n x^{n-1}\right) \\
& \lim _{x \rightarrow-\infty} \frac{g(x)}{x^{n}}=\alpha \neq \infty .
\end{aligned}
$$

The particular case given by $\lim _{x \rightarrow-\infty}(g(x) / x)=0$ is interesting. Indeed we have, for $\delta>0$

$$
\lim _{x \rightarrow-\infty} f\left(x,-\frac{\delta}{2} x\right)>\delta
$$

Then the assumption

$$
f(x, y)>\delta, \quad \text { if } x<-a, y>a
$$

implies the hypothesis $\left(5^{\prime}\right)$. This result is known [5] when $g(x)$ is a bounded function for $x$ negative.

REMARK 2.2. - We just note that the hypothesis $\left(5^{\prime}\right)$ may be replaced by the hypothesis

$$
\lim _{x \rightarrow+\infty} f(x, e-g(x))>g^{\prime}(x)+1
$$

Also in this case one may consider the observation of previous Remark 2.1.

REMARK 2.3. We consider the following autonomous equation

$$
\ddot{x}+f(x, \dot{x}) \dot{x}+g(x)=0 \text {. }
$$

For this equation we may give a result analogous with Theorem 2. More precisely one may prove that the autonomous system

$$
\left\{\begin{array}{l}
\dot{x}=y \\
\dot{y}=-f(x, y) y-g(x)
\end{array}\right.
$$

has (in the phase-plane) at least one stable limit cycle. This is equivalent to the existence of a nontrivial periodic solution of equation (2.4).

THEOREM 3. - Let $f: \boldsymbol{R}^{2} \rightarrow \boldsymbol{R}, g: \boldsymbol{R} \rightarrow \boldsymbol{R}$ such that

(1) $f$ and $g$ are continuous and locally lipschitz continuous functions;

(2) $x g(x)>0$ if $x \neq 0$ 
(3) $f(0,0)<0$ and there exist $a, M>0$ such that

$$
\begin{aligned}
f(x, y)>-M & \text { if } x<a, y>a \\
& x>-a, y<-a .
\end{aligned}
$$

There exists $\varepsilon>0$ such that

(4) $f(x, y)|y|+|g(x)| \geqslant \varepsilon$ if $|x|>a$

(5) $\lim _{x \rightarrow-\infty} f(x,-g(x))>g^{\prime}(x)+1$

$$
\text { (or } \left.\left(5^{\prime}\right) \lim _{x \rightarrow+\infty} f(x,-g(x))>g^{\prime}(x)+1\right) \text {. }
$$

Then there exists a non trivial periodic solution of equation (2.1).

Proof. - The proof of this theorem follows immediately based on the previous observations. We note that

a) The origin is the only stationary point of system (2.5) and it is a repulsive stationary point.

b) Arguing as in Theorem 2 it is possible to produce a bounded trajectory for system (2.5).

The Poincaré-Bendixson theorem now implies that system (2.5) has at least one stable limit cycle.

Thus theorem 3 is proved.

We observe that the assumption

$$
f(x, y)|y|+|g(x)|>0 \quad|x|>a
$$

cannot replace the hypothesis (4), because in this case a trajectory of system (2.5) may have an horizontal asymptote.

3. - Let us briefly examine the results we have obtained and let us compare them with the results obtained by Gaetano VHLART [5] as his are the most general among the works considered. Theorem 1 extends theorem 1 of the above-mentioned work: indeed the hypotheses (4) improve on the hypotheses

$$
\begin{array}{ll}
f(x, y) y+g(x) \geqslant E+\delta & \text { if } x>a, \quad y \geqslant 0 \\
f(x, y) y+g(x) \leqslant e-\delta & \text { if } x<-a, \quad y \leqslant 0 .
\end{array}
$$

This improvement is in line with the classical results. 
Levinson [1], indeed, has put forward the following hypotheses on the function $g(x)$

$$
\begin{aligned}
& \lim _{|x| \rightarrow+\infty}|g(x)|=+\infty \\
& x g(x)>0 \quad|x|>a \\
& \lim _{x \rightarrow \infty} \frac{g(x)}{\int_{0}^{x} g(S) d S}=0 .
\end{aligned}
$$

Subsequent papers have little by lettle improved on such hypotheses: those at (4) of theorem 1 imply the hypotheses

$$
\begin{cases}g(x) \geqslant E & \text { if } x>a \\ g(x) \leqslant e & \text { if } x<-a .\end{cases}
$$

Which improves on the results already noted, and it does not seem possible to improve on them any further with the same technique since otherwise it will be impossible to avoid the possible existence of a horizontal asymptote in the construction of region $V$.

One may use an analogous argument under hypotheses, (3) and (5) which are an improvement on the hypotheses suggested in [5]

$$
\begin{array}{lcl}
f(x, y) \geqslant \delta>0 \quad & \text { if } x>a, & y<-a \\
& x<-a, & y>a \\
f(x, y) \geqslant-M & \text { if }|x|<a, & |y|<+\infty \\
& x>a, & 0<y<a \\
& x<-a, & -a<y<0 .
\end{array}
$$

Even a result of this kind concords with the classical results becanse Levinson supposes

$$
\begin{array}{lll}
f(x, y) \geqslant \delta>0 & \text { if }|x|>a, & |y|>a \\
f(x, y)>-M & |x|<a, & |y|<+\infty
\end{array}
$$

and such a hypothesis has been gradually improved on.

Theorem 2 may be regarded as an extension of a result due to Gaetano VILLARI [5], the applications of which are interesting when $g(x)$ is bounded, but which gives few indications otherwise. 
If, finally, we consider the equation

$$
\ddot{x}+f(x) \dot{x}+g(x)=e(t)
$$

the results of theorems 1 and 2 permit both assumption (3.1) and the hypothesis relative to $f(x)$

$$
f(x) \geqslant \delta>0 \quad \text { if }|x|>a .
$$

Let us compare such hypotheses with the results obtained by MawHin [6], REISSIG [8], [9] and MaRTELLI [10].

In these papers other than continuity, no hypotheses on $f(x)$ are required.

Other hypotheses are

$$
\left\{\begin{array}{l}
\int_{\delta}^{x} e(S) d S=0 \\
x g(x) \geqslant 0 \quad \text { if }|x|>a .
\end{array}\right.
$$

We may observe that the assumptions (3.3) are for the most part equivalent to those at (3.1).

Indeed if $e(t)$ does not have mean value zero we put

$$
\int_{0}^{T} e(S) d S=I
$$

One should consider the function

$$
\hat{e}(t)=e(t)-I / T
$$

which turns out to be $T$-periodic and with mean value zero. It follows that

$$
\begin{array}{ll}
g(x) \geqslant I / T & \text { if } x>a \\
g(x) \leqslant I / T & \text { if } x<-a .
\end{array}
$$

On the other hand, it is not possible to rule out the hypothesis that

$$
\int_{0}^{r} e(S) d S=0 \text { maintaining the hypothesis that } x g(x)>0
$$

if $|x|>a$, as may be verified by the following example: 
Example 3.1. - Let $g: \boldsymbol{R} \rightarrow \boldsymbol{R}$ and $e: \boldsymbol{R} \rightarrow \boldsymbol{R}$ be such that

$$
\begin{aligned}
& g(x)=x \quad \text { if }|x| \leqslant 1 \\
& \frac{1}{x} \quad \text { if }|x| \geqslant 1 \\
& e(t)=\sin t+3 .
\end{aligned}
$$

Let us consider the equation $\ddot{x}+f(x) \dot{x}+g(x)=e(t)$. It may be verified that no matter how one takes $f(x)$ continuous, no periodie solution exists.

The result is immediate if one examines the system

$$
\begin{aligned}
& \dot{x}=y \\
& \dot{y}=-f(x) y-g(x)+e(t) .
\end{aligned}
$$

With respect to the function $g(x)$ one may observe that the hypotheses are more restrictive because

$$
\begin{gathered}
\lim _{|x| \rightarrow \infty} \sup \frac{g(x)}{x}<4 \pi^{2} \\
\left(\lim _{|x| \rightarrow \infty} \frac{g(x)}{x}=0 \text { in }[6]\right)
\end{gathered}
$$

is required.

In conclusion with respect to the function $f(x)$ the hypothesis (3.2) is not required, but the behaviour of $g(x)$ as $x \rightarrow \infty$ is constrained by that of $x$.

Finally we observe that theorems 1 and 2 provide information on boundedness of solutions for $t>0$ and give a limitation for the periodic solutions, because they must obviously be situated in the region $V$, but the method used in [6], [8], [9], [10] is valid even in the case of functions from $\boldsymbol{R}^{n}$, to $\boldsymbol{R}^{n}$ and in a more general situation.

\section{Appendix.}

Proof of LEMma 1. - We suppose that the positive half-trajectory of system (1.5) which passes through the point $P_{0}$ at the time $t_{0}-h$ does not cross the $x$-axis in a time less than $2 h$ otherwise the lemma is trivial.

At the time $t_{0}+h$ such a trajectory passes through the point having coordinates $x_{0}+2 h\left(y_{0}-\delta h\right)$ and $y_{0}-2 \delta h$.

At the time $t_{0}-h+T$, based on the definition of function $v(t)$, the trajectory passes through the point having coordinates $x_{0}+2 h^{2} \delta+T\left(y_{0}-2 \delta h\right)$ and $y_{0}-2 \delta h$. 


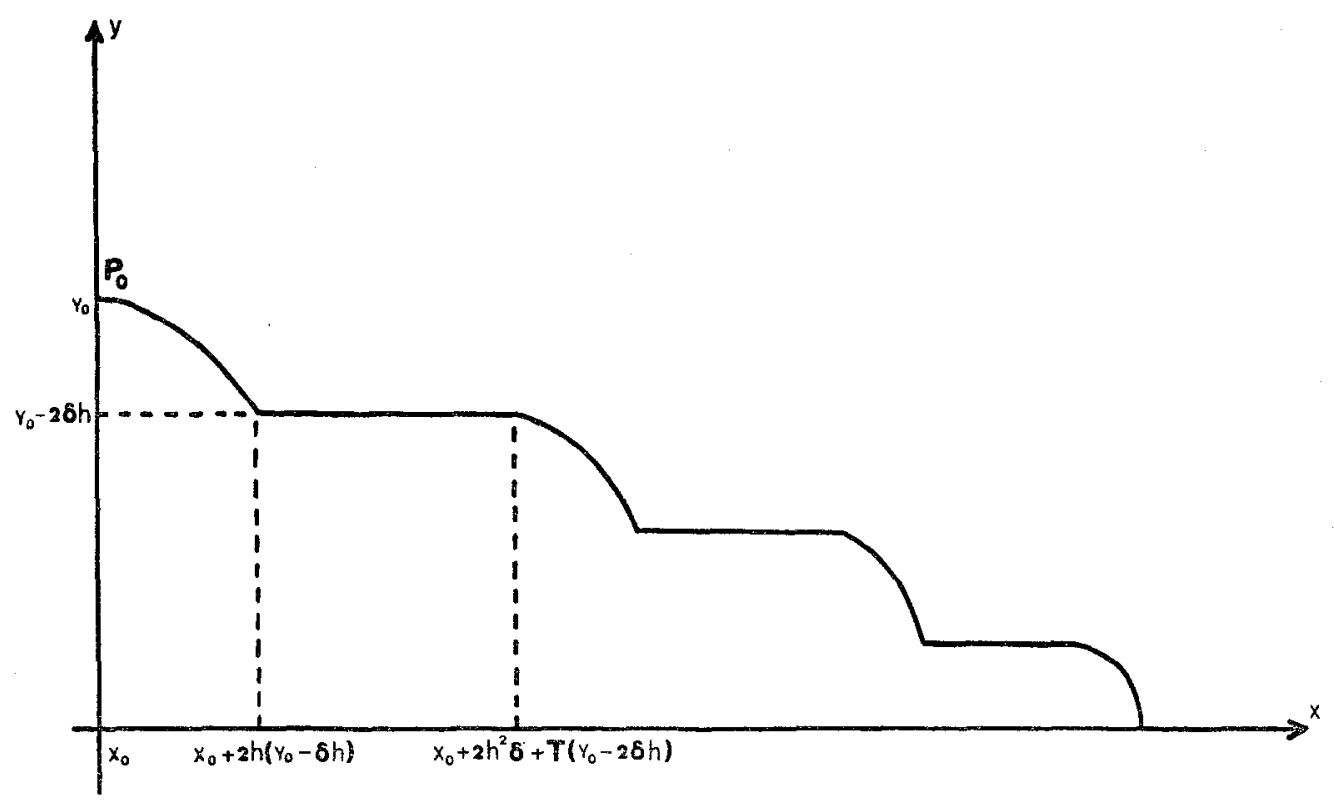

Fig. 4.

The function $v(t)$ is $T$-periodic; hence for every period $T$ the ordinate of the trajectory of system (1.5) decreases by $2 \delta h$. Let $n$ be the smallest integer such that

$$
\begin{gathered}
y_{0}<n 2 \delta h \\
n=\left[\frac{y_{0}}{2 \delta h}+1\right]
\end{gathered}
$$

Thus we have

$$
x_{1}-x_{0}<\frac{1}{2} \frac{y_{0}^{2}}{\delta}+\left[\frac{y_{0}}{2 \delta h}+1\right] \cdot(T-2 h)\left(y_{0}-2 \delta h\right)
$$

now we replace $\delta$, namely

$$
x_{1}-x_{0}<\frac{2 y_{0}^{2}}{E-e}+\left[\frac{2 y_{0}}{h(E-e)}+1\right] \cdot(T-2 h)\left(y_{0}-\frac{1}{2} h(E-e)\right) \text { Q.E.D. }
$$

Proof of LEmma 2. - We consider the negative half-trajectory of system (1.6) which passes through the point $D$ at the time $\hat{t}$.

This trajectory crosses the axis $x=x_{C}$ at a point $\hat{C}$ and the axis $x=x_{B}$ at a point $\hat{B}$.

The point $\hat{C}$ has ordinate $\hat{a}$, while the point $\hat{B}$ has ordinate $\hat{a}_{1}$ and it is $\hat{a}>a$, $\hat{a}_{1}>a_{1}$. 
Recalling (1.3), if we compare systems (1.5) and (1.6) it follows that $\left(\hat{a}_{1}-a\right)$ and $\left(\hat{a}_{1}-a_{1}\right)$ depend on $\left(x_{D}-x_{C}\right)$ and $\left(x_{D}-x_{B}\right)$ respectively, but they do not depend on $x_{C}$ or $x_{B}$.

A consequence of system $(1.6)$ is

$$
y^{\prime}=-f(x, y)-\frac{g(x)-\varphi(t)}{y}
$$

We have

$$
y^{\prime}=-f(x, y)-\frac{g(x)-\varphi(t)}{y} \leqslant-f(x, y)-\frac{g(x)-E}{y}
$$

If we suppose $f(x, y)>-M$ in the region bounded by $x>x_{\sigma}, 0 \leqslant y \leqslant \hat{a}$ from

$$
\int_{D \hat{c}} y^{\prime} d x=\hat{a}
$$

if follows

$$
\int_{s_{0} s_{1}} \hat{y}^{\prime} d x<\hat{a}+2 M\left(x_{D}-x_{C}\right)+\frac{|e|}{a_{1}}\left(x_{D}-x_{C}\right) .
$$

We note that with respect to the function $f(x, y)$ the hypothesis of boundedness in the region above mentioned is made for simplicity. In fact if $f(x, y)$ has no lower bound in this region, we may replace $f(x, y)$ with $f(x, y)>-M$.

In this case the assumption,

$$
f(x, y) y+g(x) \geqslant E \quad \text { if } x>a \quad y \geqslant 0
$$

which is in hypothesis (4), is still valid.

Consider the system

$$
\left\{\begin{array}{l}
\dot{x}=y \\
\dot{y}=-\hat{f}(x, y) y-g(x)+\varphi(t)
\end{array}\right.
$$

From a comparison with the system (1.6) it follows that the positive half-trajectory of system (A.2) which passes through the point $\hat{C}$ in the meantime, crosses the $x$-axis at a point $\hat{D}$ having coordinates $x_{\hat{D}}$ and 0 , with $x_{o}<x_{\hat{D}}<x_{D}$. Since $\left(x_{\hat{D}}-x_{o}\right)<$ $<\left(x_{D}-x_{C}\right)$, arguing in the same way we have the same statement.

Now we prove that

$$
\hat{y}^{\prime}-y^{\prime} \geqslant \frac{2}{3} k
$$

if $x>a, y \geqslant z>a, \hat{y}>a$. 
This follows from hypothesis (5) and assumption (1.4).

In fact, from

$$
\hat{y} \geqslant a_{1} \geqslant y \geqslant a
$$

we have

$$
\begin{aligned}
\hat{y}^{\prime}-y^{\prime}=f(x-\hat{y}) & +f(x, y)-\frac{g(x)}{\hat{y}}+\frac{e}{\hat{y}}+\frac{g(x)}{y}-\frac{\varphi(t)}{y} \geqslant \\
& \geqslant k-\frac{g(x)}{\hat{y}}+\frac{e}{\hat{y}}+\frac{g(x)-E}{y} \geqslant k-\frac{g(x)}{\hat{y}}+\frac{e}{\hat{y}}+\frac{g(x)}{\hat{y}}-\frac{E}{\hat{y}}=k-\frac{E-e}{\hat{y}} \geqslant \frac{2}{3} k .
\end{aligned}
$$

From (A.3) we have

$$
\int_{s_{1} \mathbf{s}_{2}} \hat{y}^{\prime} d x<\hat{a}_{1}-\hat{a}-\frac{2}{3} k\left(x_{0}-x_{B}\right)
$$

Thus $\overline{S_{2} B}=\int_{s_{0} \mathcal{B}_{2}} \hat{y} d x<\hat{a}_{1}+\left(2 M+|e| \mid a_{1}\right)\left(x_{D}-x_{C}\right)$. Since $\left(x_{D}-x_{C}\right)<\left(x_{D}-x_{B}\right)$ and, recalling Lemma 1 and assumption (1.3), $\left(x_{D}-x_{B}\right)$ and $\hat{a}_{1}$ do not depend on $x_{0}$, Lemma 2 is proved. Q.E.D.

Proof of LEmma 3. - We consider the negative half-trajectory of system (1.5) which passes through the point $B$ at the time $t_{0}-h$. This trajectory crosses at the time $\vec{t}<t_{0}-h$ the axis $x=b$ at a point $A$ having coordinates $b$ and $y_{A}$, with $y_{A} \geqslant a_{1}$.

The negative half-trajectory of system (1.6) which passes through the point $B$ at the time $t_{0}-h$ crosses the axis $x=b$ at a point $\hat{A}$ having coordinates $b$ and $y_{\hat{A}}$, with $y_{\hat{A}} \geqslant y_{A}$.

Assume $a_{1}>1$, which clearly is not restrictive.

Based on (1.3) we have

$$
y_{A} \leqslant \hat{y}_{\hat{A}}<y_{A}+\frac{k}{3}\left(x_{B}-b\right)
$$

The line $\hat{y}(x)$ which passes through the point $S_{0}$ crosses the axis $x=b$ in a point $S_{3}$.

By a possible integration of assumption (A.3) in the interval $\left[b, x_{B}\right]$, based on assumptions (1.8) and (A.1), we have

$$
\hat{y}\left(x_{B}\right)-a_{1}-\left|\hat{y}(b)-y_{\hat{A}}\right| \geqslant \frac{2}{3} k\left(x_{B}-b\right)
$$

recalling (A.4) it follows

$$
\hat{y}\left(x_{B}\right)-a_{1}-\left|\hat{y}(b)-y_{A}\right|>\frac{k}{3}\left(x_{B}-b\right)
$$


Lemma 2 may be invoked to state that

$$
\lim _{x_{B} \rightarrow+\infty}\left(y_{A}-\hat{y}(b)\right)=+\infty
$$

This means that when $x_{B}$ is big enough the line $S_{2} S_{3}$ crosses the arc of trajectory $B A$.

Therefore, recalling assumptions (A.3) and (1.3),

$$
y_{\Delta}>\hat{y}(b)
$$

In the light of the meaning of the line $S_{0} S_{3}$ Lemma 3 is proved. Q.E.D.

\section{REFERENCES}

[1] N. Levinsos, On the existence of periodic solutions for second order differential equations with a foreing term, J. Math. Phys., 22 (1943), pp. 41-48.

[2] C. LANGENHOP, Note on Levinson's exirtence theorem for forced periodic solutions of a second order differential equation, J. Math. Phys., 30 (1951), pp. 36-39.

[3] Z. Opuax, Démonstration d'un théorème de N. Levinson et C. Langenhop, Ann. Math. Pol., 7 (1960), pp. 241-246.

[4] R. REISSIG, Über die Existenz periodischer Lösungen für Differentialgleichungen 2. Ordnung mit einem Störungsglied, Math. Nachrichten, 14 (1956), pp. 341-348.

[5] G. VILLARI, Criteri di esistenza di soluzioni periodiche per una classe di equazioni differenziali del secondo ordine non lineari, Ann. Mat. Pura Appl., 65 (1964), pp. 153-166.

[6] J. MAwhin, An extension of a theorem of A. C. Lazer on forced nonlinear oscillations, J. Math. Anal. Appl., 40 (1972), pp. 20-29.

[7] A. C. LAzER, On Schauder's fixed point theorem and forced second order nonlinear oscillations, J. Math. Anal. Appl., 21 (1968), pp. 421.425.

[8] R. REISsig, Extension of some results coneerning the generalized Lienard equation, Ann. Mat. Pura Appl., 104 (1975), pp. 269-281.

[9] R. ReIssig, Schwingungssätze für die Verallgemeinerte Lienardsche Differentialgleichung, Math. Abh. Hamburg, 44 (1975), pp. 45-51.

[10] M. MaRTELLI, On forced nonlinear oscillations, J. Math. Anal. Appl., 69 (1979), pp. $496-504$. 